\title{
Accurate Lattice Energies of Organic Molecular Crystals from Periodic Turbomole Calculations
}

\author{
Hannes Konrad Buchholz, ${ }^{1,2}$ and Matthias Stein ${ }^{2}$ \\ Correspondence to: Dr Matthias Stein (E-mail: matthias.stein@mpi-magdeburg.mpg.de)
}

\begin{abstract}
Accurate lattice energies of organic crystals are important i.e. for the pharmaceutical industry. Periodic DFT calculations with atom-centred Gaussian basis functions with the Turbomole program are used to calculate lattice energies for several non-covalently bound organic molecular crystals. The accuracy and convergence of results with basis set size and k-space sampling from periodic calculations is evaluated for the two reference molecules benzoic acid and naphthalene. For the X23 benchmark set of small molecular crystals accurate lattice energies are obtained using the PBE-D3 functional. In particular for hydrogen-bonded systems, a sufficiently large basis set is required. The calculated lattice energy differences between enantiopure and racemic crystal forms for a prototype set of chiral molecules are in good agreement with experimental results and allow the rationalization and computer-aided design of chiral separation processes.
\end{abstract}

\section{Introduction}

Periodic density functional theory (DFT) calculations have wide applications in the field of solid-state chemistry, e.g. the computation of structural, chemical, optical, spectroscopic, vibrational and thermodynamic properties of crystalline materials. ${ }^{1}$ Recently, crystal structure prediction approaches to suggest possible crystal packings from only individual molecular structures to identify the most stable structure or to screen for other possible polymorph candidates have become feasible. ${ }^{2}$ Besides structural refinement, $a b$ initio quantum chemistry methods are shown to be able to compute the thermochemistry of crystalline solids with increasing accuracy.

The major contribution to the phase transition thermodynamics from the gas to the solid phase is the lattice energy $E_{\text {latt }}$.
Advances in computer technology and algorithm enable more and more reliable and fast calculations of the lattice energy of organic compounds. ${ }^{3}$ Nowadays, lattice energies can be calculated with DFT-based methods in the subkilojoule range as recently shown for benzene. ${ }^{4}$ Calculations of larger benchmark sets of small molecules also give an accuracy of better ${ }^{5,6}$ or equal ${ }^{7}$ to $\sim 5 \mathrm{~kJ} \mathrm{~mol}^{-1}$ compared to experimental data. This is assumed to be within the experimental uncertainty of measurements of sublimation enthalpies, ${ }^{8}$ the experimental counterpart to the computed lattice energies.

For the development of novel drug molecules, isolation and separation of chiral molecules is of critical scientific and industrial relevance since the 1992 FDA guideline requires new chiral drugs to be marked as single enantiomers. ${ }^{9}$ The separation of enantiomers from their mixtures especially difficult from the racemic (50:50) mixture - is most economically done by crystallization. Such a process design requires an accurate knowledge of the thermochemistry of the crystalline forms of the pure enantiomers 
and their mixtures. ${ }^{10}$ Sometimes, the molecular association in solution does not correspond to that in the crystal. ${ }^{11}$ Computational methods are of increasing practical use here as their accuracy is converging to reproduce small enantiopure/racemic energy differences. For example the lattice energy differences between different polymorphs of the same compound are usually below $4 \mathrm{~kJ} \cdot \mathrm{mol}^{-1}$ (in $80 \%$ of the cases), ${ }^{12}$ which is in the same range as the energy difference between homochiral and racemic crystals. ${ }^{13}$

With increasing accuracy of computational methods new fields of applications are being explored. Computed lattice energies have been used to calculate intrinsic solubilities from energies of the crystal structure. ${ }^{14}$ Lattice energy calculations have also been shown to be able to be able to resolve energy differences between crystals of the crystallized pure enantiomer and the racemate. ${ }^{15-17}$ These lattice energy differences can be calculated accurately enough to be able to predict relative solubilities and thus the eutectic composition of a compound-forming system in solution. ${ }^{18,19}$ However, it was recently shown that solely focussing on energy differences of the static lattice is not always sufficient for modelling the thermochemistry of molecular crystals at ambient temperature and in solution but thermal corrections and solubilisation need to be considered. ${ }^{19}$

The quantum chemical characterization of periodic systems can be done with many different approaches. Periodic boundary conditions are applied to describe a 3D infinite system. The description of this threedimensional systems can, in principle, be performed using plane-wave (PW) basis functions with pseudo-potentials, ${ }^{20}$ a linear combination of atom-centered Gaussian basis functions $^{21}$ (e.g. in CRYSTAL ${ }^{22}$ ), a linearized augmented plane-wave ansatz (LAPW) ${ }^{23}$ or a hybrid Gaussian and plane-wave scheme. ${ }^{24}$ For a review of computational methods see for example refs. ${ }^{1,25}$ The computational efficiency of
PW-DFT for homogeneous systems like metals and inorganic solids has recently been challenged by advances in computing hardware to enable periodic calculations with atomcentred basis functions for inhomogeneous systems such as molecular crystals. For a comparison of results from PWDFT vs. allelectron Gaussian atomic orbitals see ref. ${ }^{26}$

Information about crystals structures are most commonly obtained from singlecrystal X-ray crystallography. The unit cell is replicated in three directions to form the crystal. In $88 \%$ of the cases the number of symmetry independent molecules, $Z^{\prime}$, is smaller or equal to one ${ }^{27}$ but the absolute number, $Z$, can be significantly larger and thus affect the computational time especially when space group symmetry cannot be explored.

We here present the first evaluation of the accuracy of periodic DFT calculations with Turbomole for a set of organic molecular crystals. The use of atom-localized GTOs allows an elegant and consistent treatment of molecules and periodic system of any dimensionality on equal footing. ${ }^{28,29}$ For sparsely packed systems, here organic crystals, GTOs were shown to provide a high computational efficiency. ${ }^{28,29}$

In this work all-electron fully atomistic computations of the lattice energies of various molecular crystals are presented and compared to experimental lattice energies. Initial calculations were performed for crystals of naphthalene and benzoic acid in order to assess the sensitivity of results in terms of basis set convergence and k-point sampling. Results for the larger X23 benchmark set of molecular crystals are compared to experiment and previous computational work. For the prototype set of chiral molecules $(D, L)$-lactide, $(R, S)$ naproxen and $(R, S)$-3-chloromandelic acid (3CLMA) subtle lattice energy differences between the enantiopure and racemic crystals are calculated and compared to experimental results. 


\section{Methods}

\section{Definition of the Lattice Energy and Thermal}

\section{Corrections}

The lattice energy, $E_{\text {latt }}$, is defined as the energy needed for breaking up the static crystal lattice. More specifically it is defined as the energy difference between a static perfect infinite crystal (ideal static solid - iss) and its related ideal static gas (isg) of non-interacting molecules in their lowest energy conformation both at $0 \mathrm{~K}$ (eq. (1) where $\mathrm{Z}$ is the number of molecules in the unit cell)

$$
E_{\text {latt }}=\frac{E^{i s s}}{Z}-E^{i s g}
$$

For a comparison to experimental sublimation enthalpies at finite temperature, thermal corrections to the static crystal have to be added. The correction terms are comprised of the zero-point vibrational energy and thermal corrections between $0 \mathrm{~K}$ and the reference temperature. The zero-point vibrational energy difference between the ideal solid and gas ( $\Delta E_{Z P E}=E_{Z P E}^{g}-E_{Z P E}^{S}$ ) is the first term. In combination with the integral of the difference in heat capacities ( $\Delta C_{P}=C_{P}^{g}-C_{P}^{s}$ ) between gas and crystal the calculated sublimation enthalpy at the reference temperature is obtained by introducing $\Delta H_{\text {corr }}$ (eq. (2))

$$
\Delta H_{\text {subl }}=-E_{\text {latt }}+\Delta H_{\text {corr }}=-E_{\text {latt }}+\Delta E_{Z P E}+\int_{0 K}^{T} \Delta C_{P} d t
$$

\section{(2)}

A simplified expression to model the thermal correction to the lattice energy, $\Delta H_{\text {corr }}$, is the molecule and crystal structure-independent $2 R T$-approximation which is often used in computational chemistry (see eq. (3)). ${ }^{3}$

$$
\Delta H_{\text {subl }}=-E_{\text {latt }}-2 R T
$$

It is based on the assumption that zero-point vibrational energy differences between the crystal and gas can be neglected $\left(\Delta E_{Z P E}=0\right)$ and that molecular vibrations are identical in the crystal and in the gas phase. It additionally assumes that intermolecular vibrations can be treated at the high-temperature limit. Then, the heat capacity of the solid can be approximated by $C_{P}^{S}=6 R .^{3}$ For the ideal gas, translational ( $\left.\mathrm{C}_{\mathrm{P} \text {, trans }}^{\mathrm{g}}=5 / 2 R\right)$ and rotational $\left(\mathrm{C}_{\mathrm{P} \text {, rot }}^{\mathrm{g}}=3 / 2 R\right)$ degrees of freedom are considered which gives a thermal correction of $\Delta H_{\text {corr }}=(5 / 2 R-3 / 2 R-$ $6 R)=-2 R T$.

\section{Computational Details}

For the computation of lattice energies, $E_{\text {latt, }}$ periodic DFT calculations are performed using Turbomole ${ }^{30}$ version 7.1. ${ }^{31}$ The periodic 'riper' module is an implementation of Kohn-Sham DFT using atom-centred Gaussian-type basis functions to model periodic systems in one to three dimensions. A detailed description of its implementation is provided in refs. ${ }^{32-35}$ Since space group symmetry cannot be exploited, the computational effort is directly related to the number of atoms per molecules as well as the total number of molecules, Z, within the unit cell (see eq. 1 ). The largest system, $(R, S)$ naproxen, has 248 atoms in the unit cell with total of 4888 contracted basis functions (see below).

For all calculations the "def2" basis sets by Ahlrichs and coworkers ${ }^{36}$ were used. In this work the performance of three different GGAs is compared, the BP86 ${ }^{37,38}$, the BLYP ${ }^{38,39}$ and the $\mathrm{PBE}^{40}$ density functionals. The latter and the closely related hybrid PBEO functional are frequently used for periodic calculations of organic crystals. ${ }^{41}$ Furthermore, for some cases the B97-D functional was tested which was shown to be superior in comparison to the other pure GGA functionals. ${ }^{42}$ Dispersion interactions were considered through the Grimme $^{43}$ correction in all calculations. This is essential for obtaining reliable lattice energies of molecular crystals. ${ }^{44}$ All calculations were performed with the default $m 3$ grid but increased to $m 5$ in case of difficulties in SCF 
convergence. The energy difference was found to be negligible.

\section{Molecular Crystals}

Periodic DFT calculations of benzoic acid, naphthalene and the six enantiopure and racemic crystals from $(D, L)$-lactide, $(R, S)$ naproxen and $(R, S)$-3CIMA started from experimental crystal structures from crystallographic information files (CIF) from the Cambridge Structural Database (CCSD). Fractional coordinates were transformed into molecular coordinates using MERCURY V2.4. ${ }^{45}$ All unit cell parameters are given in Tables S1 to $\mathrm{S} 3$ in the ESI.

For benzoic acid two different crystal structures from CCSD entries BENZACO ${ }^{46}$ and the later refined $\mathrm{BENZACO}^{47}\left(\mathrm{Z}^{\prime}=0, Z=4, P 21 / \mathrm{c}\right)$ were used which only differ marginally in their unit cell parameters. For naphthalene, also two different structures from NAPHTAO4 ${ }^{48}$ and NAPHTA $23^{49}\left(Z^{\prime}=0, Z=2, P 21 / a\right)$ with only small differences in unit cell parameters were used to assess the energy difference between crystals.

For $(S)$ - and $(R S)$-lactide the NAHNOZ ${ }^{50}\left(Z^{\prime}=3\right.$, $Z=12, P 212121)$ and $B^{\prime C V I S}{ }^{51}\left(Z^{\prime}=1, Z=4 P 21 / C\right)$ structures were retrieved, respectively. For the pure enantiomer of 3-chloromandelic acid, $(S)$ 3 CIMA, the TUYBIA ${ }^{11}\left(Z^{\prime}=2, Z=4, P 21\right)$ and for the racemic molecular compound $(R, S)$-3CIMA the FIZPELO3 $^{11}\left(Z^{\prime}=1, Z=4, P 21 / c\right)$ crystal structures were used. Even though, $(S)$-3CIMA was shown to have a 2:1 disorder in the phenyl ring orientation only the pre-dominant (S)-3CIMA orientation was used. The $(S)$ - and $(R, S)$ naproxen crystal structures were taken from the COYRUD ${ }^{52}\left(Z^{\prime}=1, Z=2, P 21\right)$ and PAPTUX $^{17}$ $\left(Z^{\prime}=1, Z=8, P b c a\right)$ entries.

Initial crystal and gas phase structures of the X23 benchmark set were taken from Reilly ${ }^{53}$ and unit cell parameters from Otero-de-la-Roza. ${ }^{7}$ For hexamine and succinic acid CCDC structures HXMTAMO $09^{54}$ and SUCACBO $2^{55}$ were used.

\section{Naphthalene and benzoic acid benchmark calculations}

Accurate lattice energies were calculated for the two well-characterized, crystallized organic molecules naphthalene and benzoic acid as an initial benchmark. The two compounds differ in the type of intermolecular interaction that dominates. In case of benzoic acid, cyclic hydrogen-bonds between the carboxylic acid groups form strong dimeric structures. ${ }^{56}$ In contrast, the intermolecular energies of naphthalene in the crystal are dominated by weak van-der-Waals interactions. ${ }^{57}$ Experimental sublimation enthalpies were measured extensively for both crystalline compounds and result in approximately 50 published values between 1925 and today. ${ }^{58}$ The sublimation enthalpy in eq. (2) is a function of temperature. Data have been measured at different temperature (ranges) or even lack information about this essential parameter. Here, the ten most recent experimental sublimation enthalpies with specified temperature have been used to extrapolate from the experimental temperature to zero Kelvin using integrals of experimental solid state thermodynamics ${ }^{59,60}$ and calculated ideal gas heat capacities. Three popular density functionals were used (BP86, B3LYP and MO6) in combination with the def2-TZVP basis set (see Table 1). The difference between those was used as the standard deviation. Raw sublimation enthalpy data were extrapolated to standard $298 \mathrm{~K}$ to obtain $\Delta H_{\text {sublexp }}(298 \mathrm{~K})$. Additional enthalpic corrections, $\Delta H_{\text {corr, }}$ and zero-point energy corrections, $\Delta E_{Z P E}$, were then added to obtain the experimental lattice energy, $E_{\text {latt,exp }}$ (see Table 1). Assuming that the vibrational lattice and molecular modes are

\section{Results and Discussion}




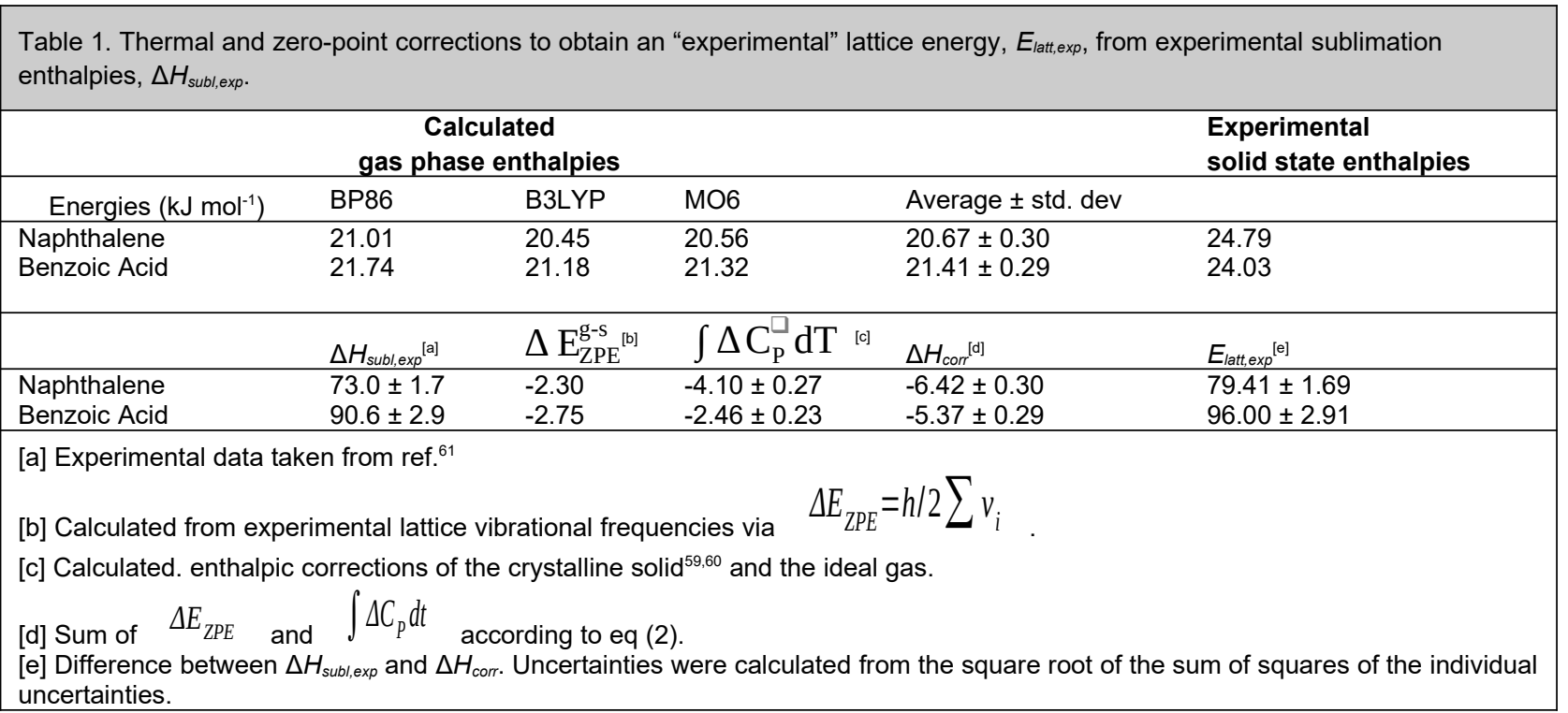

decoupled, $\Delta E_{Z P E}$ in eq. (2) can be derived from the lattice phonon modes as differences in molecular vibrations of isolated and crystallized compound would cancel out. In very flexible systems, this assumptions may not always hold. ${ }^{19}$ Experimental vibrational lattice frequencies were used to calculate $\Delta E_{Z P E}$ $\Delta E_{Z P E}=h / 2 \sum v_{i}$. This ZPE is in excellent agreement with calculated ZPEs for naphthalene ${ }^{62}$ and benzoic acid. ${ }^{63,64}$ When using the $2 R T$-approximation from eq. (3), $E_{\text {latt,exp }}$ is underestimated by 0.7 to $1.4 \mathrm{~kJ} \mathrm{~mol}^{-1}$ for benzoic acid and naphthalene, respectively.

For naphthalene and benzoic acid crystals, an exhaustive $k$-point sampling was performed from $k=1$ to $k=21$ in order to assess the convergence of lattice energies. This was done using uniform $k$-points in all three directions and no significant change for $k>3$ was observed (see ESI Table S4 for details). Hence, subsequent lattice energies were calculated using a uniform $k$-points mesh of $k=3 \times 3 \times 3$ for all calculations. Figure 1 (a) and (b) show the absolute deviations from the 'experimental' lattice energies of Table 1 . For both molecules there is a large increase in accuracy when using the larger triple-zeta (def2-TZVP) basis set

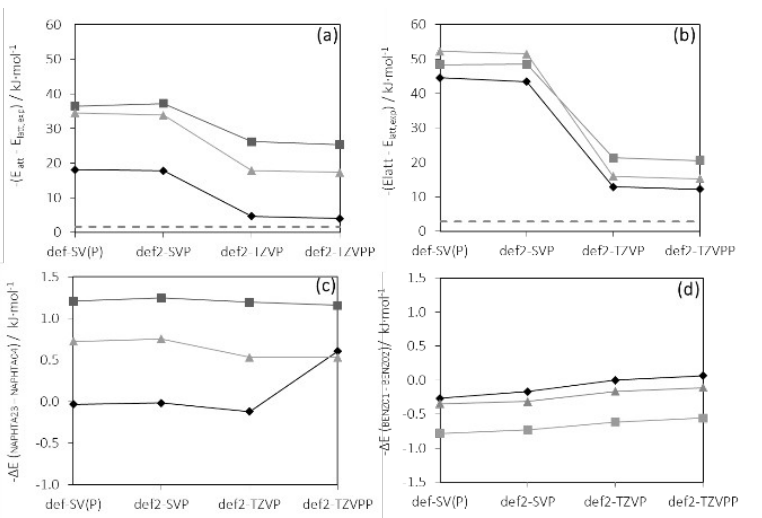

Figure 1. Basis set dependence of deviation of calculated lattice energies from experiment for (a) naphthalene (NAPHTA04) and (b) benzoic acid (BENZAC02) using BLYP ( $\mathbf{- 1}), \operatorname{BP86}(\mathbf{\Delta})$ and PBE $(\diamond)$. The dashed grey line indicates the uncertainty of the "experimental" lattice energy. (c) and (d) show the lattice energy differences between different crystal structures (NAPHTAO4 and NAPHTA23) and (BENZACO1 and BENZACO2), respectively.

compared to split-valence basis sets def-SV(P) and def2-SVP. An additional set of polarization functions for hydrogen atoms (def2-TZVPP) does not significantly improve the results. The same trend is observed for all three functionals (BLYP, BP86 and PBE). For both molecules, the PBE functional performs best whereas the BLYP 
functional leads to the largest deviation from experiment. In case of the weakly interacting naphthalene, the differences between the functionals are significantly larger than for the hydrogen-bonded benzoic acid. This is surprising as non-covalent dispersion interactions have been shown to be modelled with similar accuracy by all three functionals when combined with the D3 dispersion correction, in fact with a slightly superior performance for BP86 and BYLP over PBE was reported. ${ }^{65}$ The lattice energy dependence on basis set size is more pronounced for benzoic acid. This can be attributed to the strongly anisotropic interactions of the hydrogen bonds between the proton and the oxygen lone pair electrons. These can be more precisely modelled with more basis functions for the valence electrons as discussed later for the larger set of molecular crystals.

In order to study the sensitivity of the calculated lattice energy to the unit cell parameters, lattice energy differences between different crystals structures of the same compound were calculated: for naphthalene (NAPHTAO4 and NAPHTA23; Figure 1c) and benzoic acid (BENZACO1 and BENZAC02; Figure 1d). The differences in unit cell parameters are only very minor (see ESI Table S1 for details). For both molecules the energy differences are below $1.3 \mathrm{~kJ} \mathrm{~mol}^{-1}$ and only slightly larger for naphthalene. The energy differences are less dependent on the size of the basis set but vary slightly between three functionals. The calculated crystal energies with the PBE functional are the least sensitive with regard to unit cell parameters while the calculations with the BLYP functional are the most effected. An optimization of unit cell parameters is expected to minimize these energy differences between differently refined structures even further.

\section{Results for the X23 benchmark set}

The PBE functional was chosen for further investigations of a larger and more diverse set of molecular crystals. Recently Otero-de-la-Roza et. al. ${ }^{7}$ suggested the C21 benchmark set for studying non-covalent interactions in solids of 21 crystal structures containing two polymorphs of oxalic acid ( $\alpha$ - and $\beta$-oxalic acid). This set was extended to include hexamine (HXMTAM09 ${ }^{54}$ ) and succinic acid $\left(\mathrm{SUCACBO} 2^{55}\right.$ ) by Reilly et. al. ${ }^{53}$ and named the ' $X 23$ benchmark' set. All molecular structures within the X23 set are depicted in Figure 2 and classified by their dominant type of intermolecular interaction in the crystal.

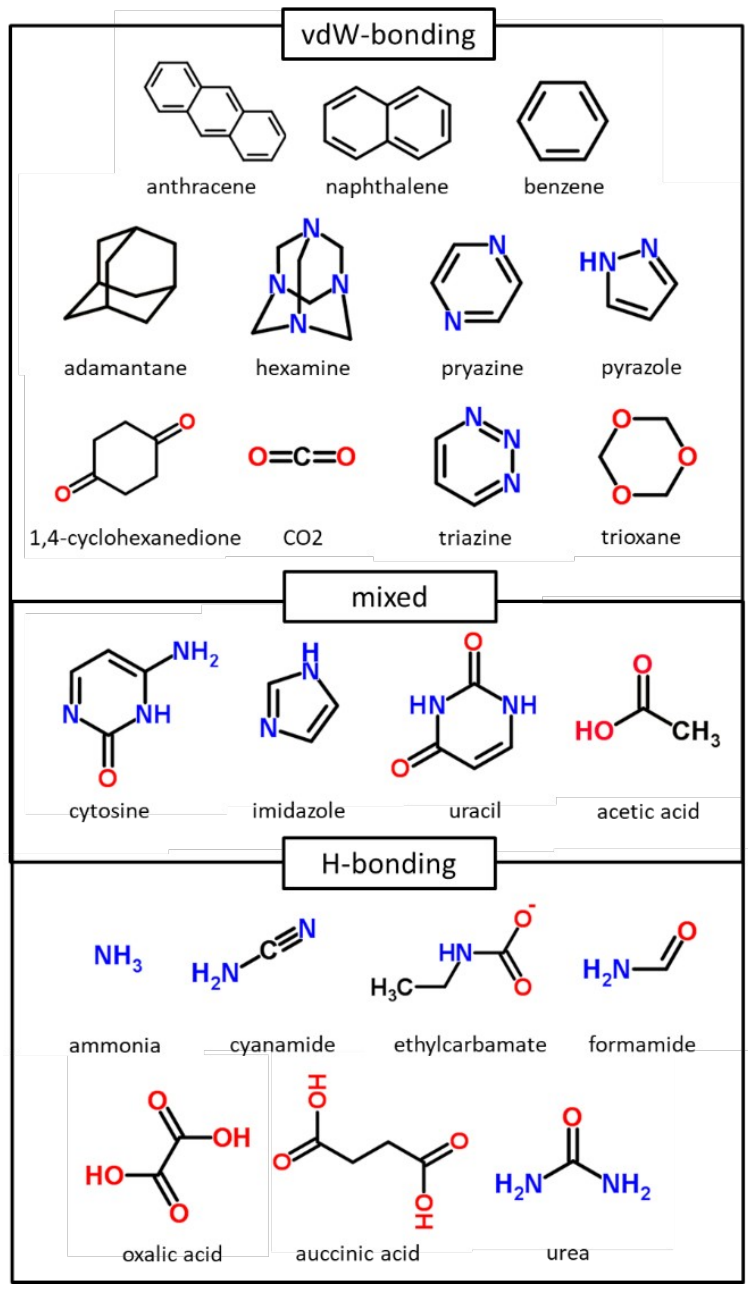

Figure 2 Molecular structures of the molecules within the X23 benchmark set for non-covalent interactions in solids. $^{753}$

Both benchmark sets refer to re-calculated 'experimental' values for the lattice energy that have been extracted from experimental 
sublimation enthalpies using different methods to model thermal and zero-point contributions to the binding energies (see eq. (2)). A comparison of the suggested thermal corrections to the frequently used structure independent $2 R T$-approxmation of eq. (3) reveals significant differences with RMSEs of 1.3 and $2.3 \mathrm{~kJ} \mathrm{~mol}^{-1}$. ${ }^{7,53}$ Here, the average of the suggested experimental lattice energies of the two studies ${ }^{7,53}$ was used while their differences give an estimate of the experimental uncertainty of $E_{\text {latt,exp. The final values and their }}$ corresponding uncertainty are summarized in Table S5 in the ESI.

Turbomole calculations were performed on the set of 23 organic molecular structures in the gas phase and in the crystal. ${ }^{7,53}$ The number of molecules within the unit cell varies between 2 $\leq Z \leq 8$ which significantly influences the computational time of the periodic calculations. The molecular structures within the unit cell and for the isolated molecules in the gas phase were optimized with the PBE-D3 density functional. The absolute deviation of our calculations from experimental lattice energies is depicted in Figure 3. For anthracene, the standard TZP basis set was used to avoid convergence difficulties with the too diffuse triple-zeta valence basis sets. The results are in excellent agreement with experiment and within the range of experimental uncertainty. There is a clear increase in accuracy when using the larger basis set TZVP in comparison to the SVP results (see Figure 3 ). We also evaluated the convergence of the lattice energy with basis set size (see ESI Fig S7). It is consistent with the previously discussed results for benzoic acid and naphthalene (see Figure 1) in that the def2TZVP basis provides reliable results. Furthermore, there is a clear tendency of the def2-SVP basis set to overestimate calculated lattice energies in particular for hydrogenbonded systems. The strong dependence on the size of the basis set to accurately model hydrogen bonding interactions is consistent with previous findings ${ }^{66,67}$ and due to a large BSSE. Results with the def2-TZVP basis set are in much better agreement with other computations (light grey area in Figure 3) and experiment (Figure 3). The light grey area indicates the standard deviation of ten different theoretical methods to calculate lattice energies. $^{7,53}$ Primary data for the average computational value are given in Table S6 in the ESI. The dark grey area gives the uncertainty of $E_{\text {latt,exp }}$ when recalculated from sublimation enthalpies via eq. (2). The deviations from experiment are largest for the extreme cases of strong $\mathrm{H}$-bonding and those dominated by vander-Waals interactions. The overall trend of our work is in agreement with previous computations and the results for the different models and the various types of molecular crystals. Our work gives a RMSE of $10.5 \mathrm{~kJ} \cdot \mathrm{mol}^{-1}$ compared to a RMSE of $9.2 \mathrm{~kJ} \cdot \mathrm{mol}^{-1}$ for an average of other computations and is well within the standard deviation of those methods (light grey shaded area). ${ }^{7,53}$

All computational methods consistently overestimate the lattice energies and show largest deviations for molecular crystals that are dominated either by mere hydrogen bonding or van der Waals interactions. We additionally show the result of the EPBEO+MBD ${ }^{36}$ method which gives the best overall representation of $E_{\text {latt,exp. }}$. The EPBEO+MBD method has an overall RMSE of $5.8 \mathrm{~kJ} \mathrm{~mol}^{-1}$ but also displays the same compound-dependent trend compared to the other computational methods. We investigated whether this effect was due to a large difference in packing for hydrogen-bonded, vander-Waals bonded or compounds with mixed intermolecular interactions in the crystal.

We emphasize this by plotting the absolute deviation of computational lattice energies from experiment (in $\mathrm{kJ} \mathrm{mol}^{-1}$ ) versus the density of the crystal $\rho_{\text {cryst }}$ in $\mathrm{g} \mathrm{cm}^{-3}$ (Figure 4 ) which ranges from $0.6 \mathrm{~g} \cdot \mathrm{cm}^{-3}$ to $2.0 \mathrm{~g} \cdot \mathrm{cm}^{-3}$. There is no obvious direct relation between the deviation of calculations from experiment and the density of the molecular crystal for neither the EPBEO + MBD or the PBE-D3/def2-TZVP calculations. 


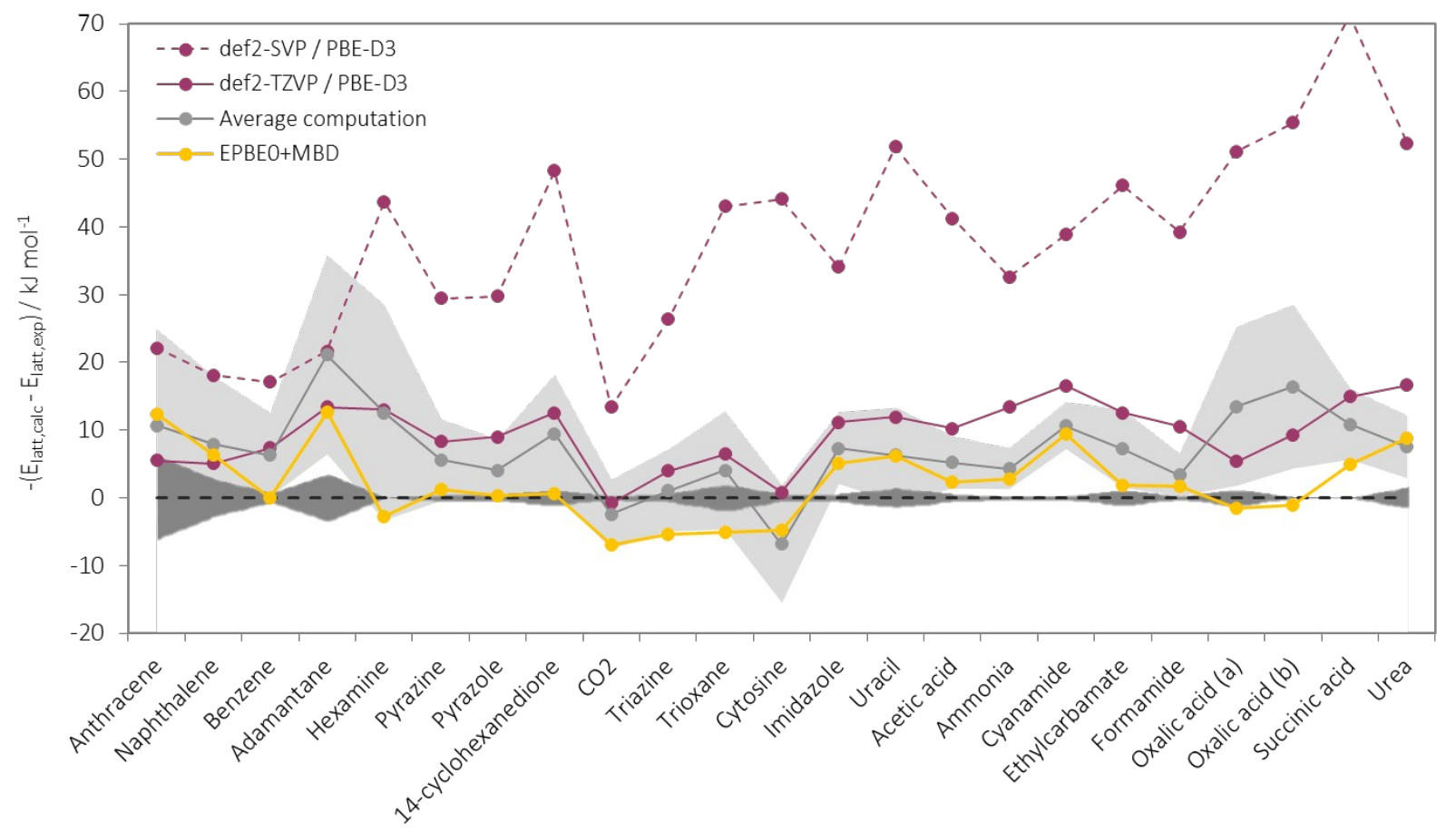

Figure 3. Lattice energies for molecules from $X 23$ benchmark set. PBE-D3 results are compared with experimental values and averaged computational values from various methods from literature ${ }^{7,53}$. The light grey area indicates the standard deviation between the ten different computational methods (see Table S6). Results from the well-performing EPBEO+MBD ${ }^{53}$ method are also included. The dark grey are indicates experimental uncertainties in obtaining lattice energies from sublimation enthalpies. 


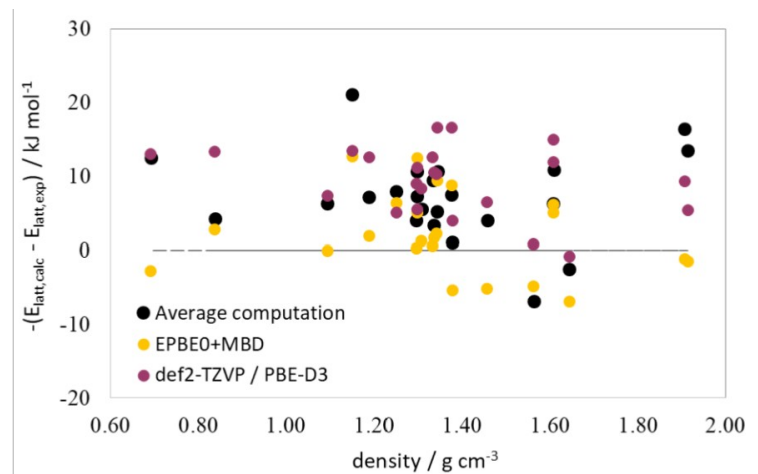

Figure 4. Error of calculated lattice energies of the X23 benchmark set and the density of the molecular crystals. PBE-D3 results are compared to the average of computational values (see Table S6) and the EPBE0+MBD calculations from ref. $^{53}$

It can be concluded that the errors in calculated lattice energies are very specific to the molecular structure and thus the type of intermolecular interaction ( $\mathrm{H}$-binding vs. vander-Waals interactions) that is dominating in the crystal rather than to the density of packing and the specific crystal structure. If the error is only dependent on the structure of the molecular compounds, such a systematic error may be advantageous when calculating energy differences between polymorphs of the same compound or different forms of chiral substances in a crystal - for example an enantiopure crystal and a racemic molecular compound. Absolute errors that are specific to each type of molecule in a lattice might cancel out when aiming for energy differences. This is shown here for a prototype set of three chiral model substances.

\section{Lattice energy differences for chiral model substances}

Until this point absolute lattice energies of molecular organic crystals and their deviations from experiment have been discussed. We here assess whether molecule-specific systematic errors in computed lattice energies would cancel when looking at differences in lattice energies between polymorphs or chiral crystals. Recent studies show that an accurate determination of lattice energy differences can be exploited to model solution phase equilibria of mixtures of enantiopure and racemic crystals. ${ }^{18,19}$ We here investigate the energy differences between the enantiopure $(e p)$ and its corresponding racemic (rac) molecular crystal for three chiral model substances, namely lactide, naproxen and 3-chloromandelic acid (3CIMA) for which there are sufficient experimental and computational data available. ${ }^{19}$ The molecular structures (see Figure 5) of the three prototypic molecules differ in their hydrogen bonding capability and in the degree of molecular flexibility. For all three molecules the periodic Turbomole calculations can be compared to previously published computations from ref. ${ }^{19}$ where DMACRYS ${ }^{68}$ and $\mathrm{HF}-3 \mathrm{c}^{69}$ were used to calculate absolute lattice energies and their differences. As reference data experimental lattice energies, $E_{\text {latt,exp, have been extracted from experimental }}$ sublimation enthalpies for lactide ${ }^{70}$ and naproxen ${ }^{71}$ using the suggested thermal and zero-point corrections from ref. ${ }^{19}$ For 3 CIMA, experimental sublimation data are not available since the compound decomposes before evaporation but absolute and relative energies can be estimated from experimental solubilities instead. ${ }^{19}$

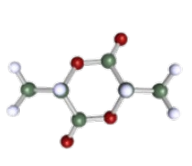

(a)

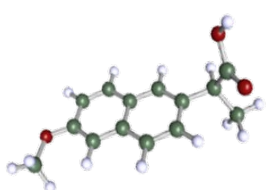

(b)

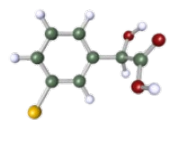

(c)
Figure 5 Lowest energy conformations of the gas phase molecular structures of (a) the rigid lactide (b) moderately flexible naproxen ${ }^{17}$ and (c) highly flexible 3CIMA. ${ }^{11}$

Table 2 gives the PBE-D3 and B97-D calculated lattice energies for racemic and enantiopure crystals of $(D, L)$-lactide, $(R, S)$-naproxen and $(R, S)$-3CIMA together with experimental lattice energies and previous calculations. ${ }^{19}$ 


\begin{tabular}{|c|c|c|c|c|c|c|c|c|c|c|}
\hline & \multicolumn{3}{|c|}{ Lactide } & \multicolumn{3}{|c|}{ Naproxen } & \multicolumn{3}{|l|}{ 3CLMA } & \multirow[t]{2}{*}{$\begin{array}{l}\text { RMSD } \\
c\end{array}$} \\
\hline & Rac. & Enant. & Diff. & Rac. & Enant. & Diff & Rac. & Enant. & Diff & \\
\hline \multicolumn{11}{|l|}{ Method } \\
\hline $\begin{array}{l}\text { def2-SVP/ } \\
\text { PBE-D3 }\end{array}$ & -144.34 & -136.75 & -7.59 & -202.87 & -194.53 & -8.34 & -187.63 & -190.63 & 3.00 & 59.3 \\
\hline $\begin{array}{l}\text { def2-TZVP/ } \\
\text { PBE-D3 }\end{array}$ & -105.35 & -99.97 & -5.38 & -158.25 & -153.67 & -4.58 & -136.10 & -136.95 & 0.85 & 14.8 \\
\hline $\begin{array}{l}\text { def2-TZVP/ } \\
\text { B97-D }\end{array}$ & -92.95 & -90.07 & -2.88 & -146.03 & -141.51 & -4.52 & -122.24 & -124.56 & 2.31 & 4.4 \\
\hline $\mathrm{HF}-3 \mathrm{c}^{19}$ & -90.10 & -87.25 & -2.85 & -135.71 & -134.26 & -1.45 & -127.43 & -126.20 & -1.23 & 5.4 \\
\hline DMACRYS ${ }^{19}$ & -86.17 & -82.64 & -3.53 & -133.48 & -125.41 & -8.07 & -105.11 & -110.40 & -5.28 & 10.7 \\
\hline Exp. & $\begin{array}{l}-97.3^{a} \\
( \pm 0.4)\end{array}$ & $\begin{array}{l}-91.8^{a} \\
( \pm 0.5) \\
\end{array}$ & $\begin{array}{l}-5.5 \\
( \pm 0.6)\end{array}$ & $\begin{array}{l}-140.8^{a} \\
( \pm 1.5)\end{array}$ & $\begin{array}{l}-134.7^{a} \\
( \pm 1.6)\end{array}$ & $\begin{array}{l}-6.1 \\
( \pm 2.2)\end{array}$ & $\begin{array}{l}-120.56^{b} \\
( \pm 1.8)\end{array}$ & $\begin{array}{l}-120.51^{b} \\
( \pm 2.4)\end{array}$ & $\begin{array}{l}-0.06 \\
( \pm 2.9)\end{array}$ & \\
\hline
\end{tabular}

[a] Experimental heat capacity corrections ${ }^{19}$ and the average of results from DMACRYS and HF-3c calculations was used. Their difference was added to the total uncertainty of $E_{\text {latt,exp. }}$.

[b] Obtained from sublimation free energies, enthalpy and entropy corrections to give $-\mathrm{E}_{\text {latt,exp }}=\Delta \mathrm{G}_{\text {subl }}-\Delta \mathrm{H}_{\text {corr }}+\mathrm{T} \Delta \mathrm{S}_{\text {subl }}$. The average of the zero-point energies from DMACRYS and HF-3c were used ${ }^{19}$ Their difference was added to the total error and is mainly responsible for the large uncertainty of $E_{\text {lattexp. }}$.

[c] Root-mean-square deviation between calculation and experimental reference for all six crystals. 
Periodic DFT calculations of the racemic (rac) of $(R, S)$-naproxen are already demanding due to the large unit cell containing $Z=8$ molecules with 248 atoms.
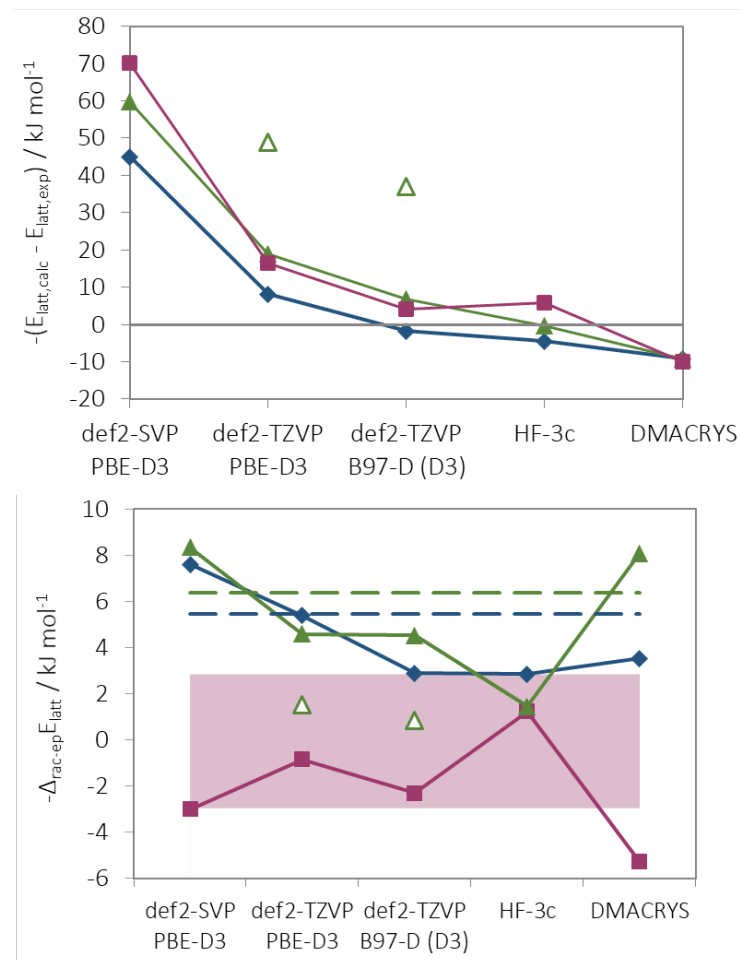

Figure 6. Calculated lattice energies for three chiral model substances: lactide ( $\diamond$, blue), naproxen ( $\boldsymbol{\Lambda}$, green) and 3-chloromandelic acid (3CIMA) (घ, magenta). (top) Deviation of calculated lattice energies from experiment for the three enantiopure crystals. (bottom) Calculated lattice energy differences between the enantiopure and racemic crystals compared to experiment (lactide blue dashed line; naproxen green dashed line; 3-chloromandelic acid magenta area). Absolute and relative energies are compared to recently reported ${ }^{19}$ calculations using DMACRYS and HF-3c. Open triangles show additional calculations for naproxen using a smaller k-point mesh of $k=1 \times 1 \times 1$.

Figure 6 (top) illustrates the deviation of calculated lattice energies from experiment for the three enantiopure crystals. There is a significant increase in accuracy of the calculated lattice energy when using a large TZVP basis set which is consistent with our results for the X23 benchmark set. The small def2-SVP had a significant RMSE of $60 \mathrm{~kJ} \cdot \mathrm{mol}^{-1}$. The PBE-D3/def2-TZVP results have an overall RMSE of $14.8 \mathrm{~kJ} \mathrm{~mol}^{-1}$. The lattice energies from B97D/def2-TZVP calculations are also in good agreement with experiment and only show an overall RMSE of $4.4 \mathrm{~kJ} \mathrm{~mol}^{-1}$ and is thus comparable to the HF-3c method with a RMSE of $5.4 \mathrm{~kJ} \mathrm{~mol}^{-1}$.

Figure 6 (bottom) gives the calculated lattice energy differences between the racemic and enantiopure crystals. Here, the results using PBE-D3 are close to the experimental value for lactide and naproxen supposingly as a result of error compensation. The effects when using a smaller (SVP) basis set are less severe for the energy differences here. The PBE-D3/def2-TZVP results are in close agreement with the results of the B97-D functional. The HF-3c method performs equally well for absolute and relative binding energies.

Since 3CIMA experimental data from sublimation vapor pressures are not available, the estimated value from experimental solubilities has a larger error due to uncertainties when determining the energy differences from experimental relative solubilities. ${ }^{19}$ However, the results for 3CIMA obtained here with the PBE-D3 and the B97-D density functionals give a consistent picture and are within the experimental uncertainty.

We also investigated the influence of a reduced $k$-point sampling for (R,S)-naproxen. The reduced sampling effects are less drastic than when using a smaller def2-SVP basis set in terms of absolute energies (Figure 6, top) but similar in magnitude for the energy differences (see Figure 6, bottom).

\section{Conclusions}

Lattice energies of organic molecular crystals were calculated using periodic Turbomole and making use of Ahlrichs' basis set. Using atom- 
centered Gaussian basis sets molecular and periodic systems can be calculated with the same approach and basis set which enables a fast and facile calculation of lattice energies. The PBE-D3 functional in combination with the def2-TZVP basis set showed the best overall agreement with experiment for the hydrogenbonded crystalline benzoic acid and pi-stacked structures of naphthalene crystals. Differences in unit cell geometries are shown to have only minor effects on the lattice energies.

In the X23 benchmark, the use of a small basis set leads to an over binding and large computational errors especially if the interactions within the crystals are dominated by one type of intermolecular interaction (either hydrogen bonding or stacking). It is the dominance of either the hydrogen bonding capability or stacking of the molecular structure in the crystal rather than a closely packed crystal that is computationally difficult to treat. Hence, it may be suggested that deviations from experimental lattice energies are less method-and density-dependent but rather due to the type of intermolecular interactions in the crystal. Lattice energy differences between enantiomeric and racemic molecular crystals, however, can be well reproduced by using periodic Turbomole calculations. In contrast to the calculation of absolute lattice energies, the calculated lattice energy differences are less sensitive to the choice of basis set and in good agreement with the experiment due to a cancellation of errors.

Periodic DFT calculations with Turbomole give accurate lattice energies for a large set of molecular organic crystals which are dominated by weak interactions such as hydrogen bonding or van-der-Waals interactions. The use the same basis set and computational approach for the molecular and the periodic system gives an elegant and straightforward way to calculate lattice energies. The cancellation of systemspecific deviations from experimental values allows the application of such computational approaches to obtain small energy differences between enantiomers and racemates and support the design of chiral separation processes for the chemical and pharmaceutical industries.

\section{Acknowledgments}

We thank the Max Planck Society for the Advancement of Science for financial support. This project was supported by the COST Action CM1402 'Crystallize'. We thank Prof. Marek Sierka (University of Jena) and Dr Uwe Huniar (Turbomole $\mathrm{GmbH}$, Karlsruhe) for technical support and scientific discussions.

Keywords: periodic DFT, chiral, molecular crystals, lattice energy.

Additional Supporting Information may be found in the online version of this article.

References

1. Hasnip, P. J.; Refson, K.; Probert, M. I. J.; Yates, J. R.; Clark, S. J.; Pickard, C. J. Philosophical Transactions Series A, 2014, 372(2011), 20130270.
2. Neumann, M. A.; van de Streek, J.; Fabbiani, F. P. A.; Hidber, P.; Grassmann, O. Nature Communications 2015, 6, 7793. 3. Gavezzotti, A. CrystEngComm 2008, 10, 367-367. 
4. $\quad$ Yang, J.; Hu, W. F.; Usvyat, D.;

Matthews, D.; Schutz, M.; Chan, G. K. L. Science 2014, 345(6197), 640-643.

5. Moellmann, J.; Grimme, S. J Phys Chem C 2014, 118(14), 7615-7621.

6. Reilly, A. M.; Tkatchenko, A. J Phys Chem Lett 2013, 4(6), 1028-1033.

7. Otero-de-la-Roza, A.; Johnson, E. R. J Chem Phys 2012, 137(5), 054103.

8. Chickos, J. S. Netsu Sokutei 2003, 30(3), 116-124.

9. US Food and Drug Administration, Chirality 1992, 4(5), 338-340.

10. Lorenz, H.; Seidel-Morgenstern, A. Angewandte Chemie International Edition 2014, 53(5), 1218-1250.

11. Hylton, R. K.; Tizzard, G. J.; Threlfall, T. L.; Ellis, A. L.; Coles, S. J.; Seaton, C. C.; Schulze, E.; Lorenz, H.; Seidel-Morgenstern, A.; Stein, M.; Price, S. L. J Am Chem Soc 2015, 137(34), 11095-11104.

12. Cruz-Cabeza, A. J.; Reutzel-Edens, S. M.; Bernstein, J. Chem Soc Rev 2015, 44(23), 86198635.

13. Gavezzotti, A.; Rizzato, S. Journal of Organic Chemistry 2014, 79, 4809-4816.

14. Palmer, D. S.; McDonagh, J. L.; Mitchell, J. B. O.; van Mourik, T.; Fedorov, M. V. J Chem Theory Comput 2012, 8(9), 3322-3337.

15. Jane Li, Z.; Ojala, W. H.; Grant, D. J. W. Journal of Pharmaceutical Sciences 2001, 90(10), 1523 -1539.

16. Perlovich, G. L.; Kurkov, S. V.; Hansen, L. K.; Bauer-Brandl, A. Journal of Pharmaceutical Sciences 2004, 93(3), 654-666.

17. Braun, D. E.; Ardid-Candel, M.; D'Oria, E.; Karamertzanis, P. G.; Arlin, J. B.; Florence, A. J.; Jones, A. G.; Price, S. L. Cryst Growth Des 2011, 11(12), 5659-5669.

18. Otero-de-la-Roza, A.; Cao, B. H.; Price, I. K.; Hein, J. E.; Johnson, E. R. Angew Chem Int Edit 2014, 53(30), 7879-7882.

19. Buchholz, H. K.; Hylton, R. K.; Brandenburg, J. G.; Seidel-Morgenstern, A.; Lorenz, H.; Stein, M.; Price, S. L. Cryst Growth Des 2017, 17(9), 4676-4686.

20. Hafner, J. Journal of Computational Chemistry 2008, 29(13), 2044-2078.
21. Towler, M. D.; Zupan, A.; Causà, M. Computer Physics Communications 1996, 98(1), 181-205.

22. Dovesi, R.; Orlando, R.; Civalleri, B.; Roetti, C.; Saunders Victor, R.; Zicovich-Wilson Claudio, M. In Zeitschrift für Kristallographie Crystalline Materials, 2005, p 571.

23. Blaha, P.; Schwarz, K.; Sorantin, P.; Trickey, S. B. Computer Physics Communications 1990, 59(2), 399-415.

24. Lippert, B. G.; Parrinello, J. H.; Michele. Molecular Physics 1997, 92(3), 477-488.

25. Dovesi, R.; Civalleri, B.; Orlando, R.;

Roetti, C.; Saunders, V. R. In Reviews in Computational Chemistry; Lipkowitz, K. B.; Larter, R.; Cundari, T. R., Eds.; John Wiley \& Sons: Hoboken, New Jersey, 2005.

26. Ulian, G.; Tosoni, S.; Valdrè, G. The Journal of Chemical Physics 2013, 139(20), 204101.

27. Brock, C. P. Acta Crystallogr B 2016, 72, 807-821.

28. Dovesi, R.; Orlando, R.; Erba, A.; Zicovich-Wilson, C. M.; Civalleri, B.; Casassa, S.; Maschio, L.; Ferrabone, M.; De La Pierre, M.; D'Arco, P.; Noël, Y.; Causà, M.; Rérat, M.; Kirtman, B. International Journal of Quantum Chemistry 2014, 114(19), 1287-1317.

29. Kudin, K. N.; Scuseria, G. E. Physical Review B 2000, 61(24), 16440-16453.

30. Furche, F.; Ahlrichs, R.; Hattig, C.; Klopper, W.; Sierka, M.; Weigend, F. Wiley Interdisciplinary Reviews-Computational Molecular Science 2014, 4(2), 91-100.

31. Turbomole; GmbH. TURBOMOLE V7.1 2016, a development of University of Karlsruhe and Forschungszentrum Karlsruhe $\mathrm{GmbH}, 1989$ 2007, TURBOMOLE GmbH, since 2007; available from http://www.turbomole.com. ; Turbomole GmbH: Karlsruhe, 2016.

32. Burow, A. M.; Sierka, M. J Chem Theory Comput 2011, 7(10), 3097-3104.

33. Burow, A. M.; Sierka, M.; Mohamed, F. The Journal of Chemical Physics 2009, 131(21), 214101.

34. Łazarski, R.; Burow, A. M.; Grajciar, L.; Sierka, M. Journal of Computational Chemistry 2016, 37(28), 2518-2526. 
35. Łazarski, R.; Burow, A. M.; Sierka, M. J Chem Theory Comput 2015, 11(7), 3029-3041.

36. Weigend, F.; Ahlrichs, R. Physical Chemistry Chemical Physics 2005, 7(18), 3297.

37. Perdew, J. P. Phys Rev B 1986, 33(12), 8822-8824.

38. Becke, A. D. Phys Rev A 1988, 38(6), 3098-3100.

39. Lee, C.; Yang, W.; Parr, R. G. Physical Review B 1988, 37(2), 785-789.

40. Paier, J.; Hirschl, R.; Marsman, M.; Kresse, G. The Journal of Chemical Physics 2005, 122(23), 234102.

41. Hoja, J.; Reilly, A. M.; Tkatchenko, A. Wiley Interdisciplinary Reviews: Computational Molecular Science 2017, 7(1), e1294.

42. Grimme, S. Journal of Computational Chemistry 2006, 27(15), 1787--1799.

43. Grimme, S.; Antony, J.; Ehrlich, S.; Krieg, H. J Chem Phys 2010, 132(15), 154104.

44. Brandenburg, J. G.; Grimme, S. Topics in current chemistry 2014, 345, 1-23.

45. Macrae, C. F.; Bruno, I. J.; Chisholm, J. A.; Edgington, P. R.; McCabe, P.; Pidcock, E.; Rodriguez-Monge, L.; Taylor, R.; van de Streek, J.; Wood, P. A. Journal of Applied Crystallography 2008, 41(2), 466-470.

46. Bruno, G.; Randaccio, L. Acta Crystallographica Section B 1980, 36(7), 17111712.

47. Feld, R.; Lehmann, M. S.; Muir, K. W.; Speakman, J. C. In Zeitschrift für Kristallographie - Crystalline Materials, 1981, p 215.

48. Ponomarev, V. I.; Filipenko, O. S.; Atovmyan, L. O. Kristallografiya 1976, 21(2), 392-394.

49. Capelli, S. C.; Albinati, A.; Mason, S. A.; Willis, B. T. M. The Journal of Physical Chemistry A 2006, 110(41), 11695-11703.

50. Belen'kaya, B. G.; Bel'skii, V. K.; Dement'ev, A. I.; Sakharova, V. I.; Chernikova, N. Y. Crystallography Reports 1997, 42(3), 449-452. 51. van Hummel, G. J.; Harkema, S.; Kohn, F. E.; Feijen, J. Acta Crystallographica Section B Structural Crystallography and Crystal Chemistry 1982, 38(5), 1679-1681.
52. Ravikumar, K.; Rajan, S. S.; Pattabhi, V. Acta Crystallogr C 1985, 41(Feb), 280-282.

53. Reilly, A. M.; Tkatchenko, A. J Chem Phys 2013, 139(2), 024705-024705.

54. Kampermann, S. P.; Ruble, J. R.; Craven, B. M. Acta Crystallographica Section BStructural Science 1994, 50, 737-741.

55. Leviel, J. L.; Auvert, G.; Savariault, J. M. Acta Crystallographica Section B-Structural Science 1981, 37(DEC), 2185-2189.

56. Gavezzotti, A. Molecular Aggregation Structure Analysis and Molecular Simulation of Crystals and Liquids; Oxford University Press: Oxford NewYork, 2007.

57. Fedorov, I. A.; Zhuravlev, Y. N.; Berveno, V. P. Physical Chemistry Chemical Physics 2011, 13(13), 5679-5686.

58. William Acree Jr. and James, S. C. Journal of Physical and Chemical Reference Data 2010, 39(4), 043101.

59. Robie, R. A.; Hemingway, B. Geological Survey Professional Paper 1972, 755.

60. Chirico, R. D.; Knipmeyer, S. E.; Steele, W. V. The Journal of Chemical Thermodynamics 2002, 34(11), 1873-1884.

61. Acree, W. J.; Chickos, J., S. Journal of Physical and Chemical Reference Data 2010, 39(4), 043101.

62. Day, G. M.; Price, S. L.; Leslie, M. J Phys Chem B 2003, 107(39), 10919-10933.

63. Li, R.; Zeitler, J. A.; Tomerini, D.; Parrott, E. P. J.; Gladden, L. F.; Day, G. M. Physical Chemistry Chemical Physics 2010, 12(20), 53295340.

64. Cysewski, P. Journal of Molecular Modeling 2015, 21(4), 83.

65. Smith, D. G. A.; Burns, L. A.; Patkowski, K.; Sherrill, C. D. The Journal of Physical Chemistry Letters 2016, 7(12), 2197-2203.

66. Brandenburg, J. G.; Alessio, M.; Civalleri, B.; Peintinger, M. F.; Bredow, T.; Grimme, S. The Journal of Physical Chemistry A 2013, 117(38), 9282-9292.

67. Riley, K. E.; Op't Holt, B. T.; Merz, K. M. J Chem Theory Comput 2007, 3(2), 407-433.

68. Price, S. L.; Leslie, M.; Welch, G. W. A.; Habgood, M.; Price, L. S.; Karamertzanis, P. G.; 
Day, G. M. Physical Chemistry Chemical Physics 2010, 12(30), 8478-8490.

69. Sure, R.; Grimme, S. Journal of

Computational Chemistry 2013, 34(19), 16721685.
70. Emel'yanenko, V. N.; Verevkin, S. P.; Pimerzin, A. A. Russian Journal of Physical Chemistry A 2009, 83(12), 2013-2021.

71. Buchholz, H.; Emel'yanenko, V. N.; Lorenz, H.; Verevkin, S. P. Journal of Pharmaceutical Sciences 2016, 105(5), 16761683. 
1 Physical and Chemical Foundations Group, Max Planck Institute for Dynamics of Complex Technical Systems, Sandtorstrasse 1, 39106 Magdeburg, Germany.

$2^{2}$ Molecular Simulations and Design Group, Max Planck Institute for Dynamics of Complex Technical Systems, Sandtorstrasse 1, 39106 Magdeburg, Germany. 